\title{
Templating effects of tetrakis(thiadiazole)porphyrazine on the structure and optical properties of copper phthalocyanine thin films
}

\author{
Keitaro Eguchi ${ }^{\mathrm{a}}$, Sandrine Heutz ${ }^{\mathrm{b}, \mathrm{c}}$ and Kunio Awaga ${ }^{\mathrm{a}}{ }^{\star}$ \\ a Department of Chemistry, Nagoya University, Furo-cho, Chikusa-ku, Nagoya 464-8602, Japan. \\ ${ }^{\mathrm{b}}$ Department of Materials, Imperial College London, Exhibition Road, London SW7 $2 A Z$. \\ ${ }^{\mathrm{c}}$ London Centre for Nanotechnology, Imperial College London, Exhibition Road, London SW7 2AZ, UK.
}

Dedicated to Professor Claudio Ercolani on the occasion of his 80th birthday

Received date (to be automatically inserted after your manuscript is submitted)

Accepted date (to be automatically inserted after your manuscript is accepted)

\begin{abstract}
Molecular templating is an attractive method to improve the crystallinity and control the molecular orientations of organic thin films. Here, we report on the templating effects of an organic ntype semiconductor, tetrakis(thiadiazole)porphyrazine $\left(\mathrm{H}_{2} \mathrm{TTDPz}\right)$, on the structure and optical absorption of a p-type semiconductor, copper phthalocyanine $(\mathrm{CuPc})$. X-ray diffraction measurements for the double layer thin films, $\mathrm{CuPc} / \mathrm{H}_{2} \mathrm{TTDPz}$, indicate a flat-on structure of $\mathrm{CuPc}$, which is replicated by the structure of the $\mathrm{H}_{2}$ TTDPz thin films, even though the CuPc thin films usually form edge-on-type thin films. The optical absorption measurements show new low-energy transitions in the templated CuPc films.
\end{abstract}

KEYWORDS: porphyrazines, molecular templating, sulfur heterocycles, flat-on structure, p-n heterostructure

*Correspondence to: Kunio Awaga; e-mail: awaga.kunio@b.mbox.nagoya-u.ac.jp; tel: +81-52-789-2487; fax: +81-52-789-2484 


\section{INTRODUCTION}

In organic electronics and optoelectronics, thin films of the organic molecules with $\pi$ conjugated electrons have received considerable attention due to their applications to organic field effect transistors (OFETs) [1], light-emitting diodes (OLEDs) [2] and photovoltaics (OPVs) [3]. Because the carriers in these devices tend to move through $\pi-\pi$ stackings, the molecular alignment in the thin films is of great importance for effective carrier transport. Roughly speaking, the molecular alignment can be classified as either an edge-on or a flat-on structure with respect to the substrate surface. The former is preferable in the OFETs, because the $\pi$ stacking can bridge the distance between the source and drain electrodes, while the latter is advantageous in the standard OLEDs and OPVs, because the $\pi$ stackings are perpendicular to the electrodes.

The molecular template effect is an attractive method for improving the crystallinity of the organic thin films and controlling their molecular orientations [4]. The thin films of 3,4,9,10-perylenetetracarboxylic dianhydride (PTCDA) were reported to grow with a flat-on structure on glass and $\mathrm{Si}$ substrates, on which the thin films of metal phthalocyanines (MPcs) also grew with a flat-on alignment, despite the fact that MPcs usually form edge-on-type thin films on these substrates, reflecting the herringbone-type molecular packings in the crystal structures of their $\alpha$ - or $\beta$ forms [5-8]. It was also reported that the use of template effects to control the molecular arrangements improved the OPV performances [6, 7]. A template effect of PTCDA was also observed for the thin film growth of cobalttetrakis(1,2,5-thiadiazole)porphyrazines (CoTTDPz); CoTTDPz formed crystalline films with a flat-on structure on the PTCDA templates, though it formed amorphous thin films without them [9].

The MTTDPz family was synthesized by Ercolani, Stuzhin and their coworkers [10,11], and in our previous works, we determined the crystal structures of the $\mathrm{M}=\mathrm{H}_{2}, \mathrm{VO}, \mathrm{Fe}, \mathrm{Co}, \mathrm{Ni}, \mathrm{Cu}$ and $\mathrm{Zn}$ derivatives [12-14]. The derivatives were found to form multi-dimensional structures that could be essentially classified into three polymorphs: the $\alpha, \beta$, and $\gamma$ forms. The crystal structure of $\mathrm{H}_{2}$ TTDPz (scheme 1a) belongs to the $\alpha$ form, which consists of a 2D molecular network formed by side-by-side $\mathrm{S}^{\delta+} \ldots \mathrm{N}^{\delta-}$ electrostatic intermolecular interactions between thiazole moieties. In this network, all the molecular planes are parallel and the $2 \mathrm{D}$ flat sheets exhibit $\pi$ stacking, perpendicular to the sheets, as in a molecular graphite [13]. We also previously studied the thin films of $\mathrm{H}_{2}$ TTDPz and their application to organic electronics, and found that $\mathrm{H}_{2} \mathrm{TTDPz}$ formed highly oriented thin films with a flat-on alignment on glass, $\mathrm{Si}$, and metal-coated glass substrates [15], which exhibited an n-type transport in OFETs [16]. In the present study, we examined the template effects of $\mathrm{H}_{2}$ TTDPz; namely, we fabricated the CuPc (scheme 1b) thin films on the $\mathrm{H}_{2} \mathrm{TTDPz}$ thin films and determined the structure and morphology of CuPc, by means of X-ray diffraction (XRD), scanning tunneling microscopy (SEM), and optical absorption spectra.

\section{EXPERIMENTAL}

$\mathrm{H}_{2}$ TTDPz was prepared as described in the literature [10]. The obtained $\mathrm{H}_{2}$ TTDPz was purified by vacuum sublimation with a continuous $\mathrm{N}_{2}$ gas flow $\left(40-50 \mathrm{ml} \mathrm{min}^{-1}\right.$ ). Commercial CuPc (Tokyo Chemical Industry Co, Ltd.) was also purified by the same method before use. The purified $\mathrm{H}_{2}$ TTDPz and CuPc were degassed for $\sim 3$ hours in home-made deposition cells in a deposition chamber (base pressure of $5 \times 10^{-5} \mathrm{~Pa}$ ) before the depositions. Then, the thin film of $\mathrm{H}_{2} \mathrm{TTDPz}(50 \mathrm{~nm})$ was deposited on a glass substrate at room temperature $(\sim 300 \mathrm{~K})$ at a deposition rate of 
$\sim 1.5 \mathrm{~nm} \mathrm{~min}{ }^{-1}$, and then CuPc was deposited on the $\mathrm{H}_{2} \mathrm{TTDPz}$ thin film at a deposition rate of $\sim 1.0 \mathrm{~nm} \mathrm{~min}^{-1}$ without air exposure. The thicknesses of the two layers were estimated from the cross-section images of SEM.

Structural and morphological characterization was performed on a RIGAKU SmartLab XRD instrument and a Hitachi SU6600 SEM. The XRD signals were recorded using $\mathrm{Cu} \mathrm{K} \alpha$ radiation $(40 \mathrm{kV}, 30 \mathrm{~mA})$ in the $2 \theta / \theta$ scan mode. The SEM measurements were performed under high vacuum conditions $\left(\mathrm{P}<10^{-3} \mathrm{~Pa}\right)$ with incident electron energies of $1.0 \mathrm{kV}$ and $5.0 \mathrm{kV}$. Optical absorption of the $\mathrm{CuPc} / \mathrm{H}_{2} \mathrm{TTDPz}$ thin films was measured on a JASCO V-570 UV-Vis-NIR spectrometer in transmission mode. The reflections at the interfaces were not corrected.

\section{RESULTS AND DISCUSSION}

To examine the template effect of $\mathrm{H}_{2} \mathrm{TTDPz}$ for $\mathrm{CuPc}$, the XRD measurements were carried out for the $\mathrm{CuPc}(l$ $\mathrm{nm}) / \mathrm{H}_{2} \operatorname{TTDPz}(50 \mathrm{~nm})$ thin films with $l=0,30$, and $60 \mathrm{~nm}$, where $l$ is the thickness of the CuPc layer. The results are shown in Fig. 1, where the XRD pattern of a CuPc thin film, deposited directly on glass, is also shown as a comparison. Note that the XRDs of the four thin films were recorded in the same intensity range and also that Fig. 1 shows the raw data without compensation for film thicknesses. The neat $\mathrm{H}_{2}$ TTDPz thin film $(l=0 \mathrm{~nm}$, Fig. 1a) exhibits a weak peak at $2 \theta=27.2^{\circ}$, which corresponds to an interplanar distance of $d=0.33 \mathrm{~nm}$. This agrees with the data in the literature [15], and could be assigned to the $(10-3)$ plane $\left(2 \theta=27.2^{\circ}\right)$ in the $\alpha$-form crystal $[12,13]$, indicating the flat-on structure of $\mathrm{H}_{2}$ TTDPz. Figure $1 \mathrm{~b}$ and $1 \mathrm{c}$ show the XRD patterns for the $\mathrm{CuPc} / \mathrm{H}_{2} \mathrm{TTDPz}$ thin films with $l=30$ and $60 \mathrm{~nm}$, respectively. They clearly indicate a peak at $27.2^{\circ}$, which increases in intensity with the increase of the thickness of the CuPc layer. This is firm evidence of the template effect of the $\mathrm{H}_{2} \mathrm{TTDPz}$ thin film for CuPc, in which the observed $d$ spacing of $0.33 \mathrm{~nm}$ is close to the face-to-face intermolecular distance $(\sim 0.34 \mathrm{~nm})$ in the $\pi$-stacking chains of $\alpha$ - and $\beta$ $\mathrm{CuPc}[17,18]$. Figure $1 \mathrm{~d}$ shows the data for the simple $\mathrm{CuPc}$ thin film, indicating the well-known peak at $2 \theta=6.7^{\circ}(d=$ $1.3 \mathrm{~nm})$, which corresponds to the (200) plane in $\alpha-\operatorname{CuPc}[6,19]$ and indicates an edge-on structure. It is notable that the $d$-spacing of $0.33 \mathrm{~nm}$ for $\mathrm{CuPc}$ in the $\mathrm{CuPc} / \mathrm{H}_{2} \mathrm{TTDPz}$ layer films would be too short to be ascribable to the interplanar distances for the herringbone $\pi$-stacking structures in $\alpha$-CuPc $(0.38 \mathrm{~nm}$ [17]) and $\beta$-CuPc $(0.48-0.50 \mathrm{~nm}$ [18, 20]). It is concluded that the flat-on structure of $\mathrm{H}_{2} \mathrm{TTDPz}$ act as a template for formation of the thin-film structure of CuPc. This is similar to the templating effects of PTCDA [6, 21, 22] and of hydrogen-terminated $\operatorname{Si}(111)$ [19], for the CuPc thin films. It is further notable that the flat-on structure of $\mathrm{CuPc}$ is maintained up to a film thickness of $60 \mathrm{~nm}$, though the XRD of this film (Fig. 1c) shows a weak peak around $6.7^{\circ}$, suggesting the slight contamination of the edge-on structure.

The surface morphology and cross-section images for the $\mathrm{CuPc}(l \mathrm{~nm}) / \mathrm{H}_{2} \operatorname{TTDPz}(50 \mathrm{~nm})$ with $l=0,30$, and $60 \mathrm{~nm}$ were examined using SEM. Fig. 2a shows the SEM images for the surface and cross-section for the $\mathrm{H}_{2} \mathrm{TTDPz}$ thin film $(l=0 \mathrm{~nm})$. They indicate the presence of nanocrystals with a diameter of $r=30-50 \mathrm{~nm}$ and a smooth surface with a height difference of $\Delta h=10 \mathrm{~nm}$. Fig. $2 \mathrm{~b}$ shows the results for the $l=30 \mathrm{~nm}$ double-layer thin film. After the deposition of $\mathrm{CuPc}$, there is little difference in the size, $r$, of the nanocrystals on the surface, but there is a clear increase in the surface roughness to $\Delta h=30 \mathrm{~nm}$. Fig. $2 \mathrm{c}$ shows the results for the $l=60 \mathrm{~nm}$ thin film, indicating a rougher surface with $\Delta h=45 \mathrm{~nm}$ and nanocrystals with diameters of 30-50 nm. This suggests a growth of the CuPc nanocrystals normal to the substrate due to the $\pi-\pi$ interactions, in contrast to CuPc with an edge-on structure, which grows in-plane to the substrate (Fig. 2d), and is consistent with the flat-on structure indicated by the XRD measurements. This strongly suggests that the lateral size of the $\mathrm{H}_{2}$ TTDPz nanocrystals in the template would govern the lateral size of the CuPc nanocrystals on the template. In other words, the lateral sizes and shapes of adlayers may also be controlled by the templates. 
To clarify the influence of the $\mathrm{H}_{2}$ TTDPz templates on the optical absorptions of the CuPc thin films, UV-vis-NIR measurements were carried out for the three thin films, $\operatorname{CuPc}(l \mathrm{~nm}) / \mathrm{H}_{2} \operatorname{TTDPz}(50 \mathrm{~nm})$ with $l=0,30$, and $60 \mathrm{~nm}$ on bare glass substrates. The results are shown in Fig. 3, together with that of a CuPc thin film (30 nm) on glass. Figure 3a displays the results for the $\mathrm{H}_{2}$ TTDPz thin film. The spectrum exhibits the three characteristic bands at around $2 \mathrm{eV}, 2.8$ $\mathrm{eV}$, and 3.5-4.0 eV, which can be assigned to the Q-band, $\mathrm{n}-\pi^{*}$ transitions and B-band, respectively [10]. The deposition of the CuPc layer on the $\mathrm{H}_{2} \mathrm{TTDPz}$ template induces the characteristic peaks, denoted as $\mathrm{S}$, A, and $\mathrm{B}$, in the Q-band region (see Figs. 3b and 3c). The transition energies are 1.4, 1.7 and $2.0 \mathrm{eV}$, respectively. Peaks A and B are considered to be ascribable to the absorptions of $\mathrm{CuPc}$, because these absorptions increase in intensity with an increase in the thickness of CuPc. Peak S at $1.4 \mathrm{eV}$ is also unique in the spectra of the templated CuPc thin films. Since the spectra has not yet been found for CuPc not only in the $\alpha$-, $\beta$-, $\gamma$-, $\delta$ - and $\varepsilon$-forms [23] but also on the PTCDA template [6], it must be caused by the unique structure of $\mathrm{CuPc}$ on the $\mathrm{H}_{2} \mathrm{TTDPz}$ template and/or an interaction between CuPc and $\mathrm{H}_{2}$ TTDPz. At this stage, it is hard to explain which of following mechanisms give rise to the spectra: charge transfer, vibronic coupling, breaking of degeneracy and/or Davydov splitting [24-26]; clearly, further investigations will be needed to clarify the origin of the characteristic bands.

\section{SUMMARY}

We examined the template effect of the $\mathrm{H}_{2} \mathrm{TTDPz}$ thin films for the growth of the CuPc layer. While the CuPc thin film prepared on the bare glass substrate formed the $\alpha$-form structure with an edge-on molecular orientation, CuPc was shown to form crystalline thin films with a flat-on molecular arrangement on the $\mathrm{H}_{2} \mathrm{TTDPz}$ templates. The SEM measurements of the $\mathrm{CuPc} / \mathrm{H}_{2} \mathrm{TTDPz}$ thin films revealed the formation of the $\mathrm{CuPc}$ nanocrystals, grown normal to the substrate and having nearly the same lateral grain size as the $\mathrm{H}_{2}$ TTDPz templates. The CuPc thin films on $\mathrm{H}_{2} \mathrm{TTDPz}$ exhibited clear differences in structure and optical absorption from the crystalline CuPc. Since $\mathrm{H}_{2} \mathrm{TTDPz}$ is an n-type organic semiconductor, the observed template effects for the p-type semiconductor, CuPc, would be applicable to the organic p-n heterojunctions for OPV cells and OLEDs.

\section{Acknowledgements}

The authors would like to thank Dr. Nakao for his technical support with the SEM measurements. A part of this work was conducted at the Institute for Molecular Science (ISM), with support from the Nanotechnology Platform (Molecule and Material Synthesis) of the Ministry of Education, Culture, Sports, Science and Technology (MEXT), Japan. This work was supported by the Japan Society for the Promotion of Science (JSPS) Core-to-Core Program and KAKENHI Grant Number 15J11122.

(a)

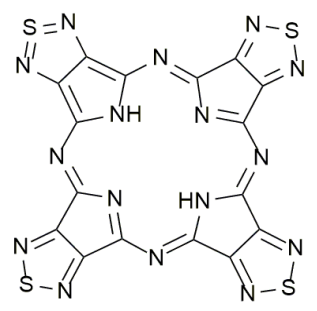

(b)

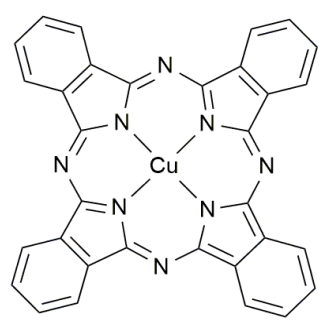


Scheme 1. Molecular structures of tetrakis(thiadiazole)porphyrazine $\left(\mathrm{H}_{2} \mathrm{TTDPz}\right)(\mathrm{a})$ and copper phthalocyanine (CuPc). (b).

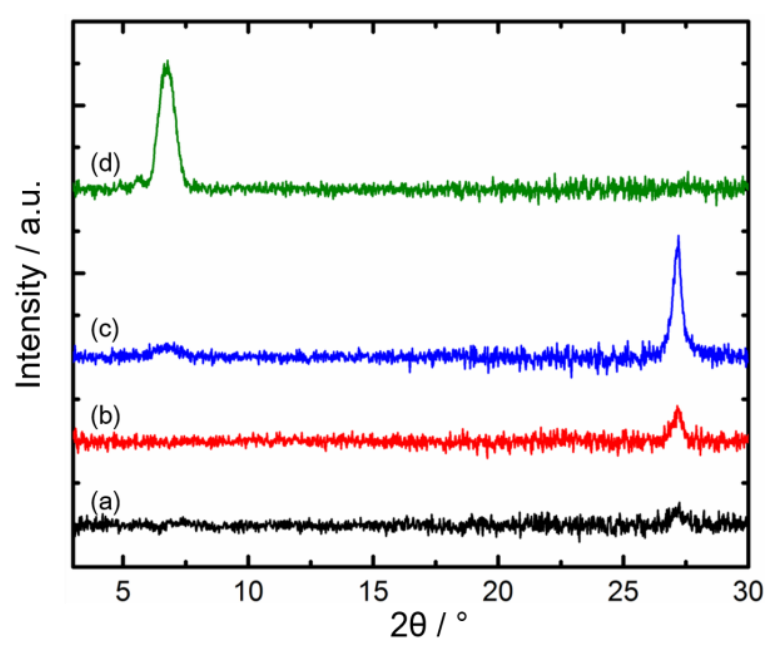

Fig. 1. XRD patterns of $\mathrm{H}_{2} \mathrm{TTDPz}(50 \mathrm{~nm}) / \mathrm{glass}$ (a), CuPc $(30 \mathrm{~nm}) / \mathrm{H}_{2} \mathrm{TTDPz}(50 \mathrm{~nm}) / \mathrm{glass}$ (b), CuPc (60 $\mathrm{nm}) / \mathrm{H}_{2} \mathrm{TTDPz}(50 \mathrm{~nm}) /$ glass $(\mathrm{c})$, and CuPc (30 nm)/glass (d).
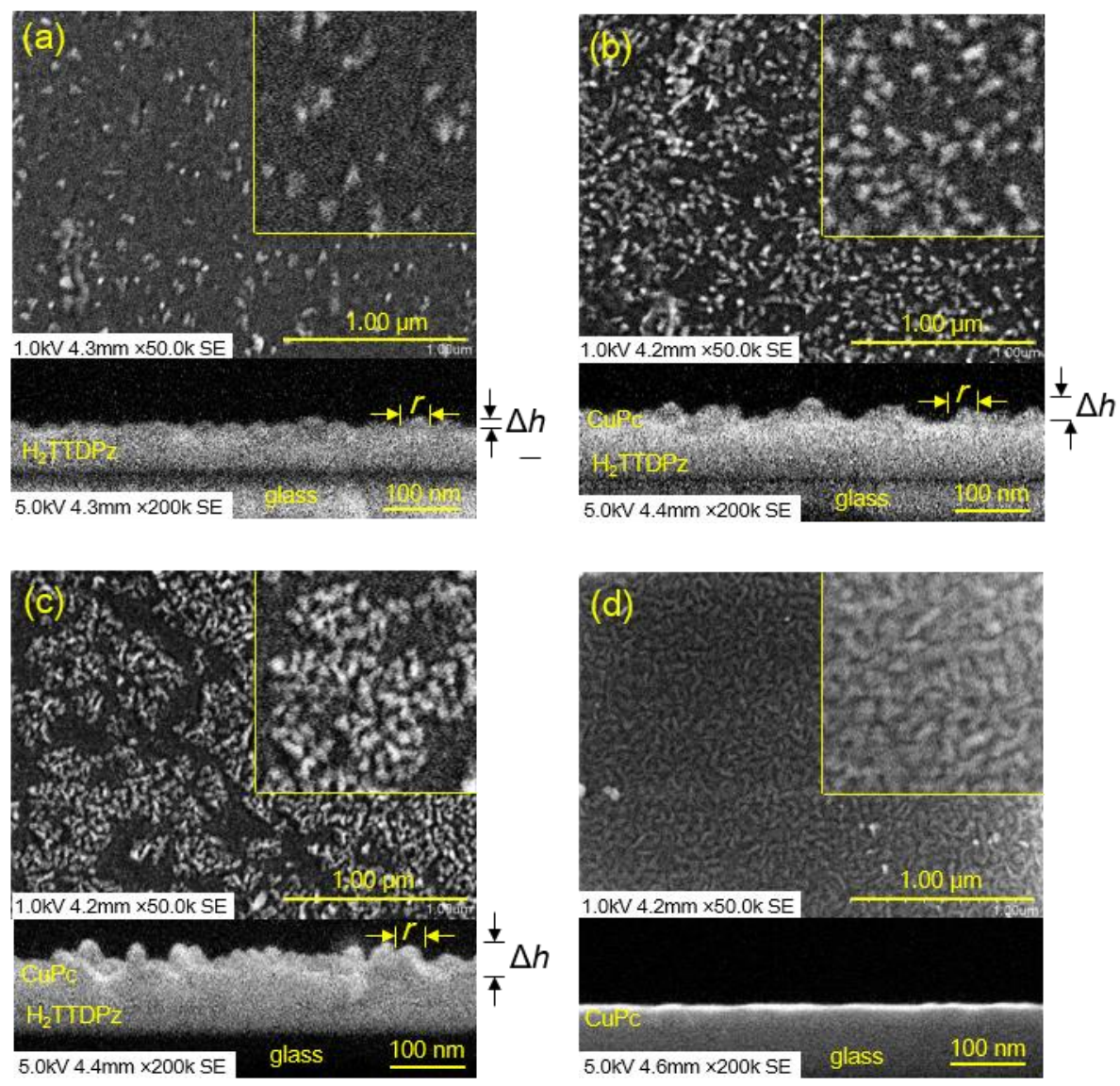

Fig. 2. SEM images of surfaces and cross-sections of $\mathrm{H}_{2} \mathrm{TTDPz}(50 \mathrm{~nm}) /$ glass (a), CuPc $(30 \mathrm{~nm}) / \mathrm{H}_{2} \mathrm{TTDPz}(50$ $\mathrm{nm}) /$ glass (b), CuPc $(60 \mathrm{~nm}) / \mathrm{H}_{2} \mathrm{TTDPz}(50 \mathrm{~nm}) /$ glass (c) and CuPc $(30 \mathrm{~nm}) /$ glass (d). Right upper panels: magnified surface images with a size of $630 \mathrm{~nm} \times 630 \mathrm{~nm}$. 


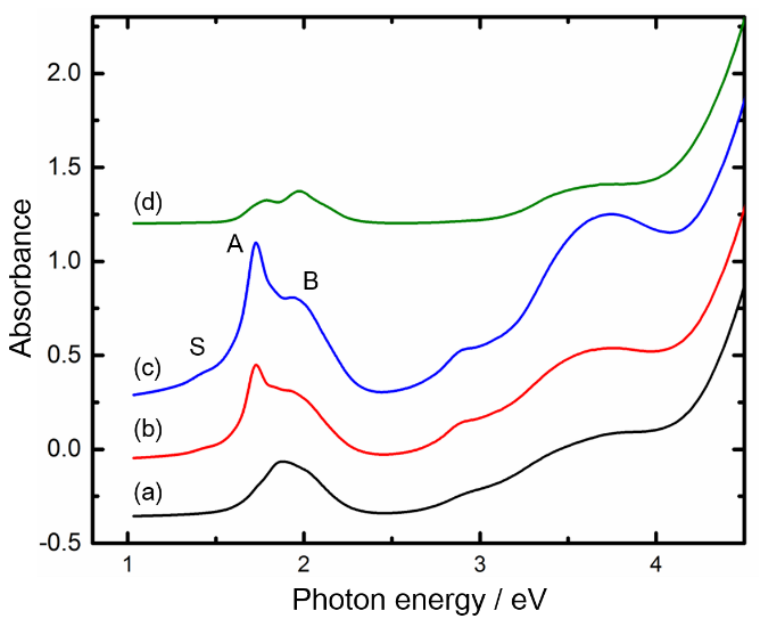

Fig. 3. UV-vis-NIR absorption spectra of $\mathrm{H}_{2} \mathrm{TTDPz}(50 \mathrm{~nm}) / \mathrm{glass}(\mathrm{a}), \mathrm{CuPc}(30 \mathrm{~nm}) / \mathrm{H}_{2} \mathrm{TTDPz}(50 \mathrm{~nm}) / \mathrm{glass}(\mathrm{b})$, CuPc $(60 \mathrm{~nm}) / \mathrm{H}_{2} \mathrm{TTDPz}(50 \mathrm{~nm}) /$ glass (c), and CuPc (30 nm)/glass (d).

\section{REFERENCES}

1. Klauk H. Chem. Soc. Rev. 2010; 39: 2643-2666.

2. Xu H, Chen R, Sun Q, Lai W, Su Q, Huang W and Liu X. Chem. Soc. Rev. 2014; 43: 3259-3302.

3. Mazzio KA and Luscombe CK. Chem. Soc. Rev. 2015; 44: 78-90.

4. Yang J, Yan D and Jones TS. Chem. Rev. 2015; 115: 5570-5603.

5. Heutz S, Cloots R and Jones TS. Appl. Phys. Lett. 2000; 77: 3938-3940.

6. Sullivan P, Jones TS, Ferguson AJ and Heutz S. Appl. Phys. Lett. 2007; 91: 233114.

7. Lassiter BE, Lunt RR, Renshaw CK and Forrest SR. Opt. Express 2010; 18: A444-A450.

8. Serri M, Wu W, Fleet LR, Harrison NM, Hirjibehedin CF, Kay CW, Fisher AJ, Aeppli G and Heutz S. Nat. Commun. 2014; 5: 3079 .

9. Eguchi K, Nanjo C, Awaga K, Tseng H-H, Robaschik P and Heutz S. Phys. Chem. Chem. Phys. 2016; 18: 17360-17365.

10. Stuzhin PA, Bauer EM and Ercolani C. Inorg. Chem. 1998; 37: 1533-1539.

11. Bauer EM, Cardarilli D, Ercolani C, Stuzhin PA and Russo U. Inorg. Chem. 1999; 38: 6114-6120.

12. Fujimori M, Suzuki Y, Yoshikawa H and Awaga K. Angew. Chem. Int. Ed. 2003; 42: 5863-5865.

13. Suzuki Y, Fujimori M, Yoshikawa H and Awaga K. Chem. Eur. J. 2004; 10: 5158-5164.

14. Miyoshi Y, Takahashi K, Fujimoto T, Yoshikawa H, Matsushita MM, Ouchi Y, Kepenekian M, Robert V, Donzello MP, Ercolani C and Awaga K. Inorg. Chem. 2011; 51: 456-462.

15. Miyoshi Y, Kubo M, Fujinawa T, Suzuki Y, Yoshikawa H and Awaga K. Angew. Chem. Int. Ed. 2007; 46: 5532-5536.

16. Miyoshi Y, Fujimoto T, Yoshikawa H, Matsushita MM, Awaga K, Yamada T and Ito H. Org. Electron. 2011; 12: $239-243$.

17. Saijo H, Kobayashi T and Uyeda N. J. Cryst. Growth 1977; 40: 118-124.

18. Qi D, Sun J, Gao X, Wang L, Chen S, Loh KP and Wee ATS. Langmuir 2010; 26: 165-172.

19. Nakamura M, Morita Y, Mori Y, Ishitani A and Tokumoto H. J. Vac. Sci. Technol., B 1996; 14: 1109-1113.

20. E J, Kim S, Lim E, Lee K, Cha D and Friedman B. Appl. Surf. Sci. 2003; 205: 274-279.

21. Heutz S, Nogueira AF, Durrant JR and Jones TS. J. Phys. Chem. B 2005; 109: 11693-11696.

22. Hong D, Do YR, Kwak HT and Yim S. J. Appl. Phys. 2011; 109: 063507.

23. Law KY. Chem. Rev. 1993; 93: 449-486.

24. Knupfer M, Schwieger T, Peisert H and Fink J. Phys. Rev. B 2004; 69: 165210.

25. Tobias B, Torsten H, Claudia M, Jens K, Michael F, Frank L, Dietrich RTZ and Georgeta S. J. Phys.: Condens. Matter 2014; 26: 104201.

26. Mali SS, Dalavi DS, Bhosale PN, Betty CA, Chauhan AK and Patil PS. RSC Advances 2012; 2: 2100-2104. 